\title{
PRIMITIVE RINGS OF INFINITE MATRICES
}

\author{
by A. D. SANDS \\ (Received 11th November 1963)
}

\section{Introduction}

E. C. Posner (5) has shown that a ring $R$ is primitive if and only if the corresponding matrix ring $M_{n}(R)$ is primitive. From this result he is able to deduce that the primitive ideals in $M_{n}(R)$ are precisely those ideals of the form $M_{n}(P)$, where $P$ is a primitive ideal in $R$. This affords an alternative proof that the Jacobson radical of $M_{n}(R)$ is $M_{n}(J)$, where $J$ is the Jacobson radical of $R$. But Patterson $(3,4)$ has shown that this last result does not hold in general for rings of infinite matrices and thus that the above result concerning primitive ideals cannot be extended to the infinite case. Nevertheless in this paper we are able to show that Posner's result on primitive rings does extend to infinite matrix rings. Patterson's result depends on showing that if the Jacobson radical $J$ of $R$ is not right vanishing then a certain matrix with entries from $J$ does not lie in the Jacobson radical of the infinite matrix ring. In the final section of this paper we consider a ring $R$ with this property and exhibit a primitive ideal in the infinite matrix ring, which does not arise, as above, from a primitive ideal in $R$. Finally the Jacobson radical of this ring is determined.

\section{Definitions and notations}

Following Patterson (3) we shall use the notation $M_{\rho}(R)$ and $M_{\rho}^{*}(R)$ for the complete ring of row-finite matrices with entries from a ring $R$ and for the complete ring of row-bounded matrices, i.e. each matrix having only a finite number of non-zero columns, with entries from $R$. We shall use $M(R)$ to denote either of these rings in cases where results hold for both rings. The corresponding column-finite and column-bounded rings of infinite matrices are denoted by $M_{\gamma}(R)$ and $M_{\gamma}^{*}(R)$. Similarly if $V$ is a module we shall use $F_{\gamma}(V)$ for the set of column vectors with entries from $V$ and $F_{\gamma}^{*}(V), F_{\rho}(V)$, $F_{p}^{*}(V)$ for the corresponding sets of column-bounded, row and row-bounded vectors.

A ring $R$ is (left) primitive if and only if it possesses a faithful irreducible (left) module, i.e. a module $V$ such that

(1) $R V \neq 0$,

(2) $V$ and 0 are the only submodules of $V$,

(3) $r V=0$, implies $r=0$. 
An ideal $P$ in a ring $R$ is primitive if and only if $R / P$ is a primitive ring. This is equivalent to $P$ being the annihilator of an irreducible $R$-module. The Jacobson radical of $R$ is the intersection of all primitive ideals of $R$ [See Jacobson (1)].

If $x$ is an element of a ring $R$ we shall denote by $x E_{i, j}$ the matrix $M(R)$ with $x$ in position $(i, j)$ and other entries zero. $L_{k}$ will denote the set of matrices in $M(R)$ whose $k$ th columns are zero and $I_{k}$ the set of matrices in $M(R)$ all of whose rows are zero, except perhaps the $k$ th row.

\section{Primitive rings}

Lemma. If $v$ is a non-zero element of a faithful irreducible module $V$ over a ring $R$ then $R v=V$.

Proof. It is easily verified that $R v$ is a submodule of $V$. Thus if $R v \neq V$ then $R v=0$. Since $v$ is not zero the submodule generated by $v$ must be $V$. Hence, if $u$ is any element of $V$, there exists $r \in R$ and an integer $n$ such that $r v+n v=u$. But $r v=0$; thus $n v=u$, and therefore $R u=R n v=n R v=0$. It follows that $R V=0$. But this contradicts (1). Therefore $R v=V$.

Theorem 1. If $R$ is a primitive ring so also is $M(R)$.

Proof. Let $V$ be a faithful irreducible $R$-module. Then $F_{y}(V)$ forms an $M(R)$-module with respect to the natural definitions of addition in $F_{\gamma}(V)$ and pre-multiplication of an element of $F_{\gamma}(V)$ by a matrix of $M(R)$.

Let $v$ be any non-zero element of $F_{\gamma}(V)$ with the non-zero element $v$ of $V$ in position $k$. Let $u$ be any element of $F_{\gamma}(V)$ with the element $u_{j}$ of $V$ in position $j$, for each $j$. By the Lemma $R v=V$. Therefore, for each $j$, there exists $r_{j} \in R$ such that $r_{j} v=u_{j}$. Let $r$ be the matrix in $M(R)$ whose $(j, k)$ th entry is $r_{j}$, for each $j$, and whose other entries are zero. Then $r v=u$. Hence $M(R) v=F_{\gamma}(V)$. It follows that $F_{\gamma}(V)$ and 0 are the only submodules of $F_{y}(V)$ and that $M(R) F_{\gamma}(V)=F_{\gamma}(V)$.

Now let $\left[r_{i j}\right]$ be a matrix of $M(R)$ such that $\left[r_{i j}\right] F_{\gamma}(V)=0$. Then it is easily seen that $r_{i j} V=0$, for each $i$ and $j$. As $V$ is faithful it follows that $r_{i j}=0$, for each $i$ and $j$. Hence $\left[r_{i j}\right]=0$ and $F_{\gamma}(V)$ is faithful.

Therefore $F_{y}(V)$ is a faithful irreducible $M(R)$-module and so $M(R)$ is a primitive ring.

The above result is proved for left primitive rings. The corresponding result may be proved for right primitive rings using $F_{\rho}^{*}(V)$, where $V$ is now a faithful irreducible right $R$-module. Similar results hold for the columnfinite and column-bounded matrices.

Theorem 2. If $M(R)$ is a primitive ring so also is $R$.

Proof. Let $W$ be a faithful irreducible $M(R)$-module. Let

$$
V=\left\{v \in W \mid L_{1} v=0\right\} .
$$

Then clearly $V$ forms a subgroup, with respect to addition, of the underlying group of $W . V$ is not zero. For, if $r$ is a non-zero matrix of $I_{1}$, then $L_{1} r=0$ 
and so $r W \subset V$. But, by (3), $r W \neq 0$. A map of $R \times V$ into $W$ is defined as follows: for each $r \in R$ and $v \in V$ let $r v=r E_{1,1} v$. As $L_{1} r E_{1,1}=0$ it follows that $r v \in V$. With this definition it is easily verified that $V$ forms an $R$-module.

Let $v$ be any non-zero element of $V$ and let $u$ be any element of $V$. By the Lemma, $M(R) v=W$. Therefore there exists a matrix $r \in M(R)$ such that $r v=u$. Let $r=r E_{1,1}+l_{1}+c_{1}$, where $r \in R, l_{1} \in L_{1}$ and $c_{1}$ has every column zero except perhaps the first and has zero in position $(1,1)$. also. Then

$$
u=r v=r E_{1,1} v+l_{1} v+c_{1} v .
$$

But $l_{1} v=0$. Thus $c_{1} v=u-r E_{1,1} v$. But $u$ and $r E_{1,1} v$ are in $V$. Hence $c_{1} v \in V$. Let $s$ be any matrix in $M(R)$. Then $s=m_{1}+d_{1}$ where $m_{1} \in L_{1}$ and $d_{1}$ has every column zero, except perhaps the first. As $c_{1} v \in V$ it follows that $m_{1} c_{1} v=0$. As $c_{1}$ has its first row zero it follows that $d_{1} c_{1} v=0$. Thus $s c_{1} v=\left(m_{1}+d_{1}\right) c_{1} v=0$. Therefore $M(R) c_{1} v=0$. It follows from the Lemma that $c_{1} v=0$. Thus $u=r E_{1,1} v=r v$. Hence $R v=V$. Therefore $R V=V$ and 0 and $V$ are the only $R$-submodules of $V$.

Suppose now that $r V=0$. Then $r E_{1,1} V=0$. Let $v$ be any non-zero element of $V$. By the Lemma $M(R) v=W$. If $w$ is any element of $W$ then, as above, there exists a matrix $f$, all of whose columns are zero except perhaps the first, such that $f v=w$. Let $f_{1}$ be the element of $R$ in position $(1,1)$ of $f$. Then $r E_{1,1} w=r E_{1,1} f v=r f_{1} E_{1,1} v=r E_{1,1} f_{1} E_{1,1} v$. But $f_{1} E_{1,1} v$ is in $V$ and $r E_{1,1} V=0$. Therefore $r E_{1,1} w=0$. Hence $r E_{1,1} W=0$. It follows from (3) that $r E_{1,1}$ is the zero matrix and so $r=0$.

Therefore $V$ is a faithful irreducible $R$-module and $R$ is a primitive ring.

Note that $I_{1} W \subset V$. But also $V=R V \subset I_{1} W$. Hence $I_{1} W=V$. However it does not seem to be easy to prove the above results starting, as Posner does, with such a definition of $V$. Of course any set $L_{k}$ could have been used instead of $L_{1}$ and so $I_{k} W=V_{k}$ is a faithful irreducible $R$-module, using the definition $r v=r E_{k, k} v$, for the map of $R \times V_{k}$ into $V_{k}$. But Posner's method of proof that $W$ is essentially a direct sum of the $V_{k}$ will not go through as infinite sums will arise in this case.

These results are for left primitive rings. Similar results may be obtained for right primitive rings and also for the rings of column finite and columnbounded matrices. The above proofs also hold for the rings of semi-infinite matrices whose rows and columns are indexed by positive integers only. The only changes necessary are to restrict $i, j$ and $k$ to be positive integers in the proofs.

Theorem 3. An ideal $P$ in a ring $R$ is primitive if and only if $M(P)$ is a primitive ideal in $M(R)$.

Proof. The rings $M(R / P)$ and $M(R) / M(P)$ are known to be isomorphic. By Theorems 1 and $2, R / P$ is a primitive ring if and only if $M(R / P)$ is a primitive ring. Thus $R / P$ is a primitive ring if and only if $M(R) / M(P)$ is a primitive ring. Hence $P$ is a primitive ideal if and only if $M(P)$ is a primitive ideal. 
This gives an alternative proof to that of Patterson (3) that the Jacobson radical $J(M(R))$ is contained in $M(J)=M(\cap P)=\cap(M(P))$, where $J=\cap P$ is the Jacobson radical of $R$, the intersections being taken over all primitive ideals of $R$.

In the case of finite matrices it follows from results of Sands (6) and Posner (5) that all prime or primitive ideals in $M_{n}(R)$ are of the form $M_{n}(P)$ where $P$ is prime or primitive in $R$. The proof in (6) involved the idea of embedding $R$ in a ring $S$ with identity and using the result that the only ideals of $M_{n}(S)$ are those of the form $M_{n}(A), A$ an ideal in $S$. More generally, Jacobson (1) has proved this last result for rings $S$ with the property that $a \in S a S$, for each $a \in S$. In the infinite case this condition remains necessary. $\dagger$ For if $a \notin S a S$ then the principal ideal generated by $a E_{i, j}$ has only elements of $S a S$ as entries in positions $(k, l)$ of its matrices, where $k \neq i, l \neq j$ and so it cannot be of the required form. But even in the case of $M_{\rho}^{*}(S)$ the condition is no longer sufficient to ensure that every ideal has the form $M_{p}^{*}(A), A$ an ideal in $S$.

For if $S$ does not satisfy the maximum condition on ideals then there exists ideals $A_{i}$ in $S$ such that $A_{n}$ is not contained in $\sum_{i=1}^{n-1} A_{i}$. Let $a_{n} \in A_{n}$, but

$$
a_{n} \notin \sum_{i=1}^{n-1} A_{i} \text {. }
$$

Consider the ideal $B=\sum_{i=1}^{\infty} M_{\rho}^{*}\left(A_{i}\right)$. Each element $a_{n}$ occurs as an entry in a matrix of $B$. But the matrix whose $(n, 1)$ th entry is $a_{n}$ and whose other entries are zero is not contained in any finite sum $\Sigma M_{\rho}^{*}\left(A_{i}\right)$ and so cannot be in $B$. Thus $B$ is not of the type $M_{\rho}^{*}(A)$.

Similar results hold in $M_{\rho}(S)$. It is not known to the author whether the conditions $a \in S a S$ and the maximum condition on ideals of $S$ are sufficient to imply that the only ideals of $M_{\rho}^{*}(S)$ are of the form $M_{\rho}^{*}(A)$, where $A$ is an ideal in $S$, or to imply that the only ideals of $M_{\rho}(S)$ are of the form

where $A, B$ are ideals in $S$.

$$
M_{\rho}^{*}(A)+M_{\rho}(B)
$$

\section{An example of a primitive ideal in $M_{\rho}(R)$}

Köthe (2) defined a ring $R$ as follows: let $R$ be generated by elements $r_{1}, r_{2}, \ldots, r_{i}, \ldots$ subject to the relations $2 r_{i}=0, r_{1}^{2}=0, r_{i+1}^{2}=r_{i}$. Then $R$ is a commutative ring. Each ideal $N_{i}$ generated by $r_{i}$ is nilpotent of index $2^{i}$. $R$ is the sum, and also the union, of these nilpotent ideals. But $R^{2}=R$, so $R$ itself is not nilpotent.

Each element $a$ in $R$ may be expressed as a polynomial in some $r_{k}$ and so in $r_{l}$ for $l \geqq k$. We denote by $e(a)$ the exponent of the non-zero term of least degree in such an expression as a polynomial in $r_{l}$, for each non-zero $a$. Then $a$ belongs to $N_{l-s}$ but not to $N_{l-s-1}$, where $2^{s} \leqq e(a)<2^{s+1}$. If $a$ and $b$ are

$\dagger$ Jacobson ((1), p. 40) mentions the sufficiency only of this condition. 
expressed as polynomials in $r_{l}$ and $e(a)<e(b)$ then we may divide $a$ into $b$ starting with the terms of least degree. The term of least degree in the remainder will eventually have degree greater than $2^{l}$. As $r_{l}^{2 l}=0$ the remainder is zero and so there exists an element $c \in R$ such that $c a=b$.

If an element $a \in R$ is such that $a \in N_{k}$ but $a \notin N_{k-1}$ then there exists an element $b \in R$ such that $a b \in N_{k}, a b \notin N_{k-1}$. For if $a$ is a polynomial in $r_{t}$ then $k=l-s$ where $2^{s} \leqq e(a)<2^{s+1}$. Taking $b=r_{1+1}$ and expressing $a b$ as a polynomial in $r_{t+1}, e(a b)=2 e(a)+1$ and so $2^{s+1} \leqq e(a b)<2^{s+2}$, giving the required result.

Consider the ideal $P$ in $M_{\rho}(R)$ given by $P=M_{\rho}^{*}(R)+\sum_{k=1}^{\infty} M_{\rho}\left(N_{k}\right)$. As the ideals $N_{i}$ form a strictly increasing chain

$$
N_{1} \subset N_{2} \subset \ldots \subset N_{i} \subset \ldots \text {, }
$$

it is clear that $P$ does not have the form $M_{\rho}^{*}(A)+M_{\rho}(B)$ where $A, B$ are ideals in $R$. We show that $P$ is the annihilator of an irreducible $M_{\rho}(R)$-module and so that $P$ is a primitive ideal.

$V=F_{\gamma}(R)$ is a module and $W=\sum_{k=1}^{\infty} F_{\gamma}\left(N_{k}\right)$ is a proper submodule of $V$. Let $v \in V, v \notin W$ and let $u$ be any element of $V$. As $v \notin W$ it follows that for each $k$ there exists a component $v_{i}$ of $v$ such that $v_{i} \notin N_{k}$. Let $u_{m}$ be the $m$ th component of $u$. Then $u_{m}$ is a polynomial in $r_{l}$ for all sufficiently large $l$. There exists $v_{i_{m}}$ such that $v_{i_{m}}$ is a polynomial in some $r_{l}$ with $e\left(v_{i_{m}}\right)<e\left(u_{m}\right)$. For if $e\left(v_{i}\right) \geqq e\left(u_{m}\right)$ for all $i$, then each $v_{i}$ belongs to the same $N_{k}$ as $u_{m}$, which is impossible. Thus there exists $c_{m} \in R$ such that $c_{m} v_{i_{m}}=u_{m}$. Let $c$ be the matrix whose $\left(m, i_{m}\right)$ th entry is $c_{m}$, for each $m$, and whose other entries are zero. Then $c v=u$. Therefore $M_{\rho}(R) v=V$ and so $W$ is a maximal submodule of $V$. Hence $V / W$ is an irreducible $M_{\rho}(R)$-module.

Let $r \in M_{\rho}^{*}(R)$ and $v \in V$. Then the only components of $v$ which occur in forming the components of $r v$ are those in positions corresponding to the finite number of non-zero columns in $\boldsymbol{r}$. These components of $v$ lie in a finite number of ideals $N_{i}$ and hence $r v \in W$. Therefore $M_{\rho}^{*}(R)$ annihilates $V / W$.

Let $s \in \sum_{k=1}^{\infty} M_{\rho}\left(N_{k}\right)$. Then $s$ is in some finite sum $\sum_{k=1}^{m} M_{\rho}\left(N_{k}\right)$. Hence for any $v \in V$, sv lies in $\sum_{k=1}^{m} F_{\gamma}\left(N_{k}\right)$ and so in $W$. Thus $\sum_{k=1}^{\infty} M_{\rho}\left(N_{k}\right)$ annihilates $V / W$.

Now let $a$ be a matrix not in $M_{\rho}^{*}(R)+\sum_{k=1}^{\infty} M_{\rho}\left(N_{k}\right)$. We construct a vector $v$ in $V$ such that $a v$ is not in $W$. Pick any non-zero row, say row $i_{1}$, in $a$. Let $a_{i_{1}, j_{1}}$ be an entry such that $a_{i_{1}, j_{1}} \in N_{k_{1}}$, but is not in $N_{k_{1}-1}$, and such that $a_{i_{1}, j} \in N_{k_{1}-1}$ for $|j|>\left|j_{1}\right|$. Let $b_{j_{1}}$ be such that $a_{i_{1}, j_{1}} b_{j_{1}} \in N_{k_{1}}$, but is not in $N_{k_{1}-1}$, and let $l_{1}$ be such that $b_{j_{1}} \in N_{l_{1}}, b_{j_{1}} \notin N_{l_{1}-1}$ and so $l_{1} \geqq k_{1}$. Not every entry $a_{i j},|j|>\left|j_{1}\right|$, can lie in $N_{l_{1}}$. For, if so, $a \in M_{\rho}^{*}(R)+M_{\rho}\left(N_{l_{1}}\right)$. Thus there exists $a_{i_{2}, j 2}$, such that $\left|j_{2}\right|>\left|j_{1}\right|, a_{i_{2}, j_{2}} \in N_{k_{2}}, a_{i_{2}, j_{2}} \notin N_{k_{2}-1}$, 
where $k_{2}>l_{1}$ and where $a_{i_{2}, j} \in N_{k_{2}-1}$ for $|j|>\left|j_{2}\right|$. Further there exists $b_{j_{2}}$ such that $a_{i_{2}, j_{2}} b_{j_{2}} \in N_{k_{2}}, \notin N_{k_{2}-1}$, where $b_{j_{2}} \in N_{l_{2}}, \notin N_{l_{2}-1}$ and $l_{2} \geqq k_{2}$. This process may be continued to produce a sequence of elements $a_{i_{m}, j_{m}}$, which occur as entries in $a$, and elements $b_{j_{m}}$ with these properties. Now let $v$ be the vector whose components are given by $v_{j_{m}}=b_{j_{m}}, v_{j}=0$ otherwise. Consider $a v$; the $i_{m}$ th component of $a v$ is $\sum_{j} a_{i_{m}, j} b_{j}$. For $j=j_{m}$ this gives $a_{i_{m}, j_{m}} b_{j_{m}}$ which is in $N_{k_{m}}$ but is not in $N_{k_{m}-1}$. For $|j|<\left|j_{m}\right|, b_{j}$ is zero or belongs to $N_{l}$ where $l_{t}<k_{m}$ and hence all such terms belong to $N_{k_{m}-1}$. For $|j|>\left|j_{m}\right|, a_{i_{m}, j}$ belongs to $N_{k_{m}-1}$ and so all such terms belong to $N_{k_{m}-1}$. Finally with $j=-j_{m}, b_{j}$ is zero. Thus every term $a_{i_{m}, j} b_{j}$, except $a_{i_{m}}, j_{m} b_{j_{m}}$, belongs to $N_{k_{m}-1}$. Hence the $i_{m}$ th term of $a v$ belongs to $N_{k_{m}}$ but not to $N_{k_{m}-1}$. As $k_{1}<k_{2}<\ldots<k_{m}<\ldots$ it follows that $a v$ belongs to no finite sum $\sum_{k=1}^{m} F_{\gamma}\left(N_{k}\right)$.

Thus $a v$ is not in $W$. Therefore $a$ is not in the annihilator of $V / W$.

Thus the annihilator of the irreducible $M_{\rho}(R)$-module $V / W$ is

$$
P=M_{\rho}^{*}(R)+\sum_{k=1}^{\infty} M_{\rho}\left(N_{k}\right)
$$

Therefore $P$ is a primitive ideal.

$P$ is in fact the Jacobson radical of $M_{\rho}(R)$. For being primitive it must contain the Jacobson radical. But as $R$ is a sum of nilpotent ideals the Jacobson radical of $R$ is $R$. Thus, by results of Patterson (3), $M_{\rho}^{*}(R)$ is contained in the Jacobson radical of $M_{\rho}(R)$. As $N_{k}$ is nilpotent so also is $M_{\rho}\left(N_{k}\right)$. Therefore $\sum_{k=1}^{\infty} M_{\rho}\left(N_{k}\right)$ is also in the Jacobson radical. Hence $P$ is contained in the Jacobson radical and so must be the radical.

In this example the radical coincides with the ideal $M_{p}^{*}(J)+\Sigma M_{\rho}(B)$ where $J$ is the Jacobson radical of $R$ and the summation is taken over the right vanishing ideals $B$ of $R$, as this ideal clearly contains $P$ but is always contained in the radical. Thus the question raised by Patterson (4) remains open as to whether or not this ideal is always equal to the radical. Nilpotent ideals are right vanishing, but the converse need not hold. It is not known to the author, however, whether the sum of all nilpotent ideals is ever strictly contained in the sum of all right vanishing ideals, i.e. whether or not the principal ideal generated by a single element $b$ in a right vanishing ideal $B$ can be non-nilpotent. Thus it is possible that the radical of $M_{\rho}(R)$ may be equal to $M_{\rho}^{*}(J)+\Sigma M_{\rho}(N)$ where the summation is taken over all nilpotent ideals $N$ of $R$, for any ring $R$.

\section{Appendix}

It has been suggested that the ring of Köthe used in Section 4 may perhaps collapse and hence collapse to zero. For if $r_{i}=r_{j}$ where $i \neq j$ it follows easily that every $r_{i}$ is zero. In this section we construct a homomorphic image of $R$ which is clearly not zero.

Let $S$ be an additive semi-group and $A$ a ring. The generalised polynomial 
ring $P(S, A)$ with coefficients in $A$ and exponents in $S$ may be defined as follows. $P(S, A)$ is the set of all maps $f$ from $S$ into $A$, such that $f(s)$ is non-zero for only a finite number of elements $s \in S$; sums and products are defined by

$$
\begin{aligned}
(f+g)(s) & =f(s)+g(s), \\
(f g)(s) & =\sum_{u+v=s} f(u) g(v),
\end{aligned}
$$

where the summation is taken over the finite number of products $f(u) g(v)$ such that $f(u)$ and $g(v)$ are both non-zero; an empty summation is taken to give zero. It is readily verified that $P(S, A)$ is a ring and that if $S$ is the set of non-negative integers then $P(S, A)$ is isomorphic to the usual polynomial ring in one variable over $A$. (By taking direct sums of semi-groups generalised polynomials rings in more than one variable may be defined similarly.)

If $I$ is an ideal in the semi-group $S$, i.e. $I+S \subset I, S+I \subset I$, then the maps of $P(S, A)$ which are zero outside $I$ form an ideal in $P(S, A)$. Clearly this ideal is isomorphic to $P(I, A)$.

Now take the special case in which $S$ is the additive semi-group of positive rationals whose denominators are non-negative powers of 2 and in which $A$ is the field of two elements. Let $I$ be the ideal consisting of those elements of $S$ which are greater than or equal to 1 . Consider the quotient ring of $P(S, A)$ modulo the ideal $B$ isomorphic to $P(I, A)$.

For each positive integer $i$ define the map $f_{i}\left(2^{-i}\right)=1, f_{i}(s)=0$ otherwise. Then $2 f_{i}=0, f_{i+1}^{2}=f_{i}$ and $f_{1}^{2}$ is the map $g$ such that $g(1)=1, g(s)=0$ otherwise and so $f_{1}^{2} \in B$. Clearly $f_{i}$ generate $P(S, A)$. Thus $P(S, A) / B$ is a homomorphic image of $R$ and clearly $P(S, A) / B$ is not zero. Note that $f_{1} f_{2} \ldots f_{n}$ is a map taking the value 1 at $1-2^{-n}$ and being zero elsewhere. Thus

and $R$ is not right-vanishing.

$$
r_{1} r_{2} \ldots r_{n} \neq 0
$$

\section{REFERENCES}

(1) N. JACobson, Structure of Rings (Amer. Math. Soc. Colloq. Publ. 37, 1956).

(2) G. KöтHE, Die Struktur der Ringe, deren Restkassenring mach dem Radikal vollständig reduzibel ist., Math. Zeitschrift, 32 (1930), 161-186.

(3) E. M. Patrerson, On the radicals of certain rings of infinite matrices, Proc. Royal Soc. Edinburgh, 65 (1961), 263-271.

(4) E. M. Patterson, On the radicals of rings of row-finite matrices, Proc. Royal Soc. Edinburgh, 66 (1962), 42-46.

(5) E. C. Posner, Primitive matrix rings, Archiv der Math., 12 (1961), 97-101.

(6) A. D. SANDs, Prime ideals in matrix rings, Proc. Glasgow Math. Assoc., 2 (1956), 193-195.

\section{Department of Mathematics QUEen's COLLEGE \\ DUNDEE}

